OPEN ACCESS

Edited by:

Lutz Walter,

Deutsches Primatenzentrum,

Germany

Reviewed by:

Stephen K. Anderson,

National Cancer Institute at Frederick,

United States

Lisbeth Guethlein,

Stanford University, United States

*Correspondence:

David Roe

roex0050@umn.edu

Specialty section:

This article was submitted to

NK and Innate

Lymphoid Cell Biology,

a section of the journal

Frontiers in Immunology

Received: 14 July 2020

Accepted: 17 September 2020

Published: 09 October 2020

Citation:

Roe $D$, Williams J, Ivery $K$, Brouckaert J, Downey N, Locklear C, Kuang R and Maiers M (2020) Efficient

Sequencing, Assembly, and Annotation of Human KIR Haplotypes.

Front. Immunol. 11:582927.

doi: 10.3389/fimmu.2020.582927

\section{Efficient Sequencing, Assembly, and Annotation of Human KIR Haplotypes}

\author{
David Roe ${ }^{1 *}$, Jonathan Williams ${ }^{2}$, Keyton Ivery ${ }^{2}$, Jenny Brouckaert ${ }^{2}$, Nick Downey ${ }^{3}$, \\ Chad Locklear ${ }^{3}$, Rui Kuang ${ }^{1,4}$ and Martin Maiers ${ }^{5}$ \\ ${ }^{1}$ Bioinformatics and Computational Biology, University of Minnesota, Rochester, MN, United States, ${ }^{2}$ DNA Identification \\ Testing Division, Laboratory Corporation of America Holdings, Burlington, NC, United States, ${ }^{3}$ Integrated DNA Technologies, \\ Inc., Coralville, IA, United States, ${ }^{4}$ Department of Computer Science and Engineering, University of Minnesota, Minneapolis, \\ MN, United States, ${ }^{5}$ Center for International Blood and Marrow Transplant Research, Minneapolis, MN, United States
}

The homology, recombination, variation, and repetitive elements in the natural killer-cell immunoglobulin-like receptor $(\mathrm{KIR})$ region has made full haplotype DNA interpretation impossible in a high-throughput workflow. Here, we present a new approach using longread sequencing to efficiently capture, sequence, and assemble diploid human KIR haplotypes. Probes were designed to capture KIR fragments efficiently by leveraging the repeating homology of the region. IDT $x G e n^{\circledR}$ Lockdown probes were used to capture 2-8 kb of sheared DNA fragments followed by sequencing on a PacBio Sequel. The sequences were error corrected, binned, and then assembled using the Canu assembler. The location of genes and their exon/intron boundaries are included in the workflow. The assembly and annotation was evaluated on 16 individuals (8 African American and 8 Europeans) from whom ground truth was known via long-range sequencing with fosmid library preparation. Using only 18 capture probes, the results show that the assemblies cover $97 \%$ of the GenBank reference, are $99.97 \%$ concordant, and it takes only 1.8 haplotigs to cover $75 \%$ of the reference. We also report the first assembly of diploid KIR haplotypes from long-read WGS. Our targeted hybridization probe capture and sequencing approach is the first of its kind to fully sequence and phase all diploid human KIR haplotypes, and it is efficient enough for population-scale studies and clinical use. The open and free software is available at https://github.com/droeatumn/kass and supported by a environment at https://hub.docker.com/repository/docker/ droeatumn/kass.

Keywords: killer-cell immunoglobulin-like receptor, assembly, DNA, haplotype, annotation, natural killer

\section{INTRODUCTION}

The protein coding killer-cell immunoglobulin-like receptor (KIR) genes span $\sim 10-16 \mathrm{~kb}$ each, with pseudogenes that are $\sim 5$ and $\sim 13 \mathrm{~kb}$. Alleles from any two genes are over $85-98 \%$ identical. Frequent recombination throughout the $\sim 70-270 \mathrm{~kb}$ haplotypes has made their order and copy number highly variable. The genes encode proteins that recognize human leukocyte antigen (HLA) and its peptide, and along with other receptors, initiate signaling pathways in natural killer (NK) cells that can lead to the release of cytokines or to the death of the target cell (infected, cancerous, 
foreign, etc.). Some of these ligand-receptor interactions stimulate the NK cell to react, while some inhibit the NK cell from reacting until the ligand is missing. NKs and their KIR receptors are essential to human health and have functional roles that impact viral infections, pregnancy, autoimmune diseases, transplantation, and immunotherapy (1-7).

Genetic interpretation of exonic-or-lower resolutions from next generation sequencing (NGS) is often ambiguous and unphased, and therefore limits precise understanding of how KIR sequences affect phenotypes. The importance of highthroughput high-resolution typing is exemplified by the fact that the genes contain extensive exonic SNP and short insertion/deletion (indel) variations which rivals that of its binding partner: HLA class I $(1,8)$. Over 300 full-length DNA and almost 1,000 protein reference alleles have been reported in IPD-KIR (9). All resolutions except haplotyping are ambiguous or require statistical phasing from few references. A cost-effective high-throughput method that could characterize all the sequences within the KIR haplotypes in cis could advance that understanding and clarify previously ambiguous and/or contradictory evidence. To date, the only approach for full haplotyping was to physically separate and amplify maternal and paternal haplotypes via fosmids for subsequent sequencing (10-14), a process whose expense has generally prohibited its use in large-scale association studies. While high-resolution haplotyping by fosmid clones or full gene by PCR is costly and inefficient, low resolution genotyping of gene presence/absence or copy number provide limited information for functional analysis and association tests.

Like much of chromosome 19, the KIR region is dense with repetitive elements, which have provided the mechanisms for its recent evolution by tandem duplication and homologous recombination events. Dozens of distinct gene-content haplotypes are seen in Europeans alone $(11,12,15,16)$. Previous reports have documented over ten distinct common haplotype structures (13). Figure 1 provides an overview of the most common haplotype structures and their informal names. KIR haplotypes are named in two halves: "c" for centromeric (i.e., proximal) and " $\mathrm{t}$ " for telomeric (i.e., distal) separated by a recombination hotspot contained in the $\sim 10 \mathrm{~kb}$ intergenic region between KIR3DP1 and KIR2DL4. Each half is also labelled " $\mathrm{A}$ " or " $\mathrm{B}$ ", designating one of two families of haplotypes, based on the gene content (17). The A family haplotype denotes haplotypes with one main gene content structure and relatively large allelic variation. The $\mathrm{B}$ family of haplotypes denotes a class of haplotypes with relatively more structural variation and less allelic variation. The haplotype named "cA01 tB01", for example, means the first (01) centromeric A region in cis (“ with the first telomeric $\mathrm{B}$ region.

It is difficult to interpret KIR haplotypes for an individual human genome given the reads from high-throughput sequencing when the structural arrangements are unknown. This is largely due to read lengths from prevailing technologies being too short to map unambiguously to the repetitive and homologous KIR genes. Even if the reads could be uniquely placed, they require statistical phasing that is difficult due to lack of phased high-resolution reference libraries. As a consequence, the reads from the KIR region are largely ignored, misinterpreted, or under-interpreted in current whole genome sequencing (WGS) studies. Therefore, the properties of the KIR region require more careful and specific interpretations than most other regions in human genome.

Here we present an approach that leverages PacBio's longread circular consensus sequence (CCS) reads to span DNA homology, and gene homology to efficiently capture $2-8 \mathrm{~kb}$ fragments of DNA. It is a workflow to capture, sequence, assemble, and annotate diploid human KIR haplotypes. And it also has broader implications to other genomic regions with variable or repetitive regions alternating with constant regions. When applied to a cohort of 8 African Americans and a cohort of 8 Europeans, the results demonstrate that every KIR gene and intergene contains constant regions that are targetable by capture probes, and that by targeting the constant regions, the variable regions can be captured and sequenced by standard PacBio workflows. Further, maximizing this paradigm shows that 18 short probe sequences can capture KIR haplotypes and allow

\begin{tabular}{|c|c|c|c|c|c|c|c|c|c|c|c|c|c|c|c|}
\hline \multirow{2}{*}{$\begin{array}{c}\text { informal } \\
\text { name } \\
\end{array}$} & \multicolumn{8}{|c|}{ proximal 'cen tromeric' regions } & intervening & \multicolumn{6}{|c|}{ distal 'telomeric' regions } \\
\hline & 3DL3 & 2DS2 & 2DL2L3 & 2DL5B & 2DS3S5 & 2DP1 & 2DL1 & 3DP1 & \begin{tabular}{|l|} 
3DP1-2DL4 \\
\end{tabular} & 2DL4 & 3DL1S1 & 2DL5B & 2DS3S4S 5 & $2 \mathrm{DS} 1$ & 3DL2 \\
\hline cA01 $\mathrm{tA} 01$ & & & E & & & 틀 & & ए & & & 는 & & E & & \\
\hline cA01 tA02 & & & & & & & & - & & & 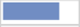 & & & & [ \\
\hline $\mathrm{cA02}$ - $\mathrm{tA} 03$ & 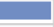 & & & & & $\square$ & $\square$ & & & & & & & & $\square$ \\
\hline $\mathrm{cA} 03 \sim \mathrm{tB} 02$ & & & & & & $\square$ & $\square$ & & & & & & & $\square$ & 는 \\
\hline $\mathrm{cB} 01 \sim \mathrm{tB} 01$ & & & & & & & & & & & & & & & \\
\hline $\mathrm{cA} 01 \sim \mathrm{tB} 01$ & 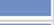 & & & & & & & L & & & ㅍ & L & $=$ & & \\
\hline cB01 tA01 & & & & & & & & L & & & & & & & \\
\hline $\mathrm{cB} 02-\mathrm{tA} 01$ & & & & & & & & 든 & & & L & & & & \\
\hline $\mathrm{cB} 03-\mathrm{tA} 01$ & & & & & & & & & & & 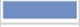 & & & & \\
\hline $\mathrm{cB} 04-\mathrm{tB} 03$ & & & & & & & & & & & & & & & \\
\hline $\mathrm{cB} 05 \sim \mathrm{tA} 01$ & $=$ & I & & & & & & & & & L & & & & \\
\hline $\mathrm{cB} 05 \sim \mathrm{tB} 01$ & & I & & & & & & & & & & & & & \\
\hline
\end{tabular}

FIGURE 1 | Common KIR haplotype structures and their names. Blue regions represent the presence of a gene or the KIR3DP1-KIR2DL4 intervening intergenic region. Some genes are partially blue to indicate their portion of a fusion allele. The first column contains the informal name for the haplotype. Each haplotype name is a combination of its two regions: centromeric (proximal) regions are preceded with a "c" and telomeric (distal) regions are preceded with a "t". Not all haplotype structures are shown. 
their unambiguous assembly. Finally, this is an efficient approach that requires no prior knowledge of the individual or references, only utilizes standard lab workflows, and is available in free and open software.

\section{MATERIALS AND METHODS}

\section{Overview}

The goal of the experiment was to create a set of capture probes and a bioinformatics workflow to efficiently assemble full KIR haplotypes from PacBio CCS reads. The experiments to capture, sequence, assemble, and annotate are depicted graphically in Figure 2. The major steps consist of

1. Design capture probes.

2. Use the probes to capture the KIR DNA fragments in vitro or in silico per individual.

3. Sequence the fragments on PacBio Sequel.

4. Error correct the sequences.

5. Bin the sequences per KIR region and gene.
6. de novo assemble all the sequences together and each gene bin separately.

7. Annotate the assembled sequences with their genes and exon/ intron locations.

\section{Step 1: Design Capture Probes}

Published KIR haplotypes sequences (36 at the time of this study) as well as all allele sequences from IPD-KIR 2.7.1 (18) were used to generate 200 candidate capture probes. The design of the 120 base probes was coordinated with a combination of automated and manual Integrated DNA Technologies (IDT) design tools, including a strain typer alignment tool and a xGen ${ }^{\circledR}$ Lockdown probe design tool. The candidate set of probes was reduced by leveraging sequence homology. First, the haplotype sequences were aligned to two KIR haplotypes (GenBank accessions GU182358 and GU182339). These two reference haplotypes, which together contain all KIR genes, were annotated via RepeatMasker $(19,20)$. The candidates were prioritized by the highest number of times each aligned to the two reference sequences but not to repetitive elements. The set was chosen

A Fragment capture \& sequencing

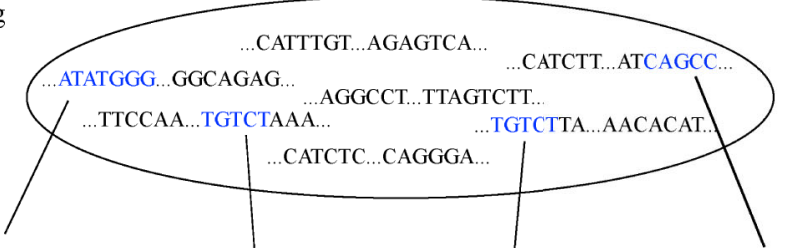

Probe 1: ATATG ...

Probe 2: TGTCT ...

Probe n: TGTCT ...

Probe 18: $\mathrm{CAGCC} \ldots$

B Error correction

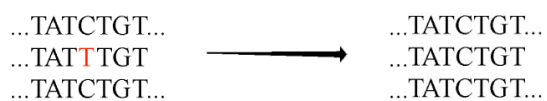

C Binning

TATCTGT

...TATCTGT.

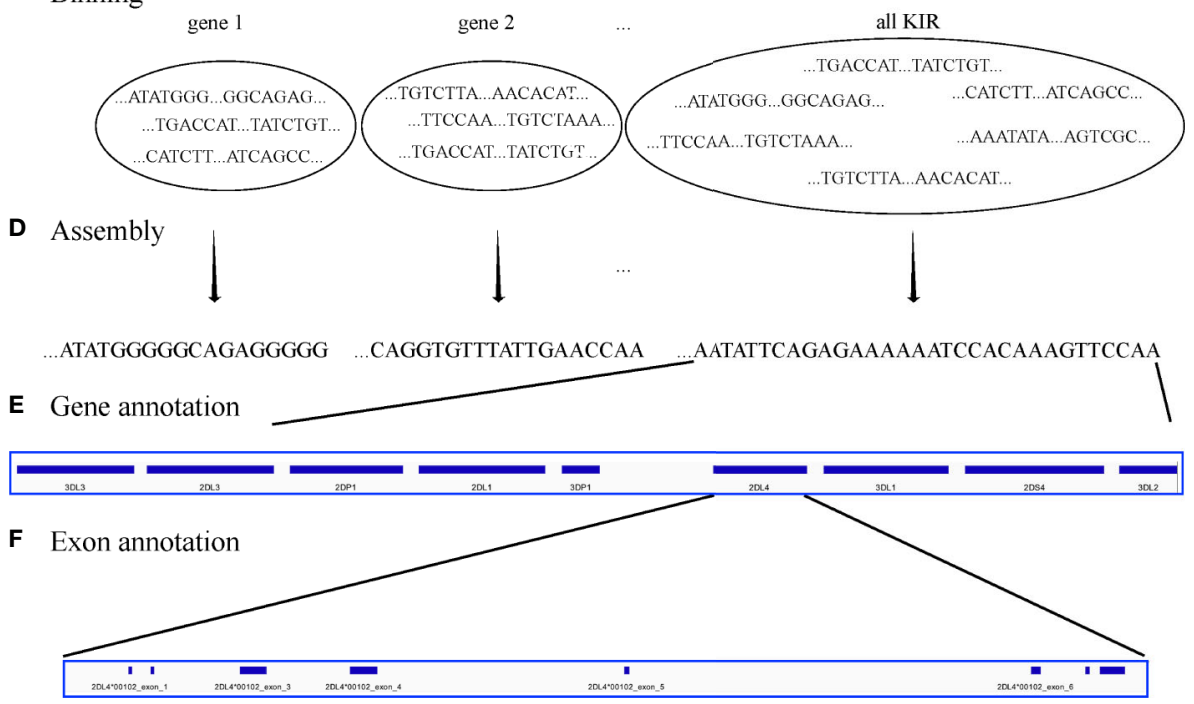

FIGURE 2 | Workflow. The workflow starts with fragment capture in vitro or in silico and PacBio assembly (A). The sequences are error corrected (B) and binned by KIR region and KIR gene (C) before de novo assembly of each bin (D). Finally, the assembled haplotigs/haplotypes are annotated by gene (E) and exon (F). 
by iteratively adding the probe with the highest alignment hit count until both reference haplotypes were covered by less than the expected average DNA fragment length of $\sim 4-5 \mathrm{~kb}$. Ultimately, experiments were conducted on the original set of 200 , a minimal set of 15 , and a refined set of 18 capture probes.

\section{Steps 2-3: Capture and Sequence the DNA Fragments per Individual}

Targeted hybridization probe capture and sequencing was performed (Figure 2A) as described using PacBio unsupported protocol PN101-388-000 (21) with the following modifications. Two $\mu \mathrm{g}$ Human Genomic DNA suspended in $200 \mu \mathrm{l}$ if Elution Buffer was sheered with Covaris G-tubes to 6-8 kb according to manufactures instructions followed by 1:1 PB AMPure bead cleanup. The target fragment size of $6-8 \mathrm{~kb}$ was chosen to maximize the ability for capture and to allow proper phasing and the generation of CCS reads, or a consensus sequence of one captured DNA fragment per sequence well. Individual specimens were then library prepped with the KAPA library prep kit (Roche) which consisted of end repair and ligation of uniquely barcoded adapters that also contained the PacBio Universal Primer sequence. After a $0.8 \mathrm{X}$ PB AMPure bead clean up samples were enriched with eight PCR cycles in a $200 \mu \mathrm{l}$ reaction using LA Taq by Takara and PacBio Universal primers followed by a 1:2 PB AMPure bead cleanup. Sample concentrations were measured on the Promega Quantus and 2 $\mu \mathrm{g}$ was size selected for greater than $2 \mathrm{~kb}$ fragments on the Blue Pippin System (Sage Sciences).

Eight multiplexed samples for Sequel sequencing were pooled at this point at $0.25 \mu \mathrm{g}$ per sample and $1.5 \mu \mathrm{g}$ of pooled size selected DNA, $5 \mu \mathrm{l}$ of $1 \mathrm{mg} / \mathrm{ml}$ Human Cot-1 DNA by Thermo Fisher, and $10 \mu \mathrm{l}$ of $100 \mu \mathrm{M}$ PacBio Universal Primer were dried in a Speed Vac with no heat. IDT $x \mathrm{Gen}^{\circledR}$ lockdown reagents and probes were used to resuspend the genomic/Cot-1/primer mixture according to manufactures instructions and incubated at $70^{\circ} \mathrm{C}$ for $4 \mathrm{~h}$ followed by IDT xGEN washes ${ }^{\circledR}$. DNA was then removed from streptavidin beads and further enriched with 15 PCR cycles using LA Taq. DNA fragments were then library prepped and sequenced eight samples per SMRT cell on the Sequel according to PacBio instructions. Raw sequence data was then demultiplexed and CCS reads generated on SmrtLink 6.0 using 99.9\% subread accuracy filter for generation.

\section{Steps 4-6: Correct, Bin, and Assemble the Sequences}

For both targeted and WGS, the fastq sequences were error corrected with LoRMA (22). The sequences were binned for onKIR and also binned per genic or intergenic region in silico (Figures 2B, C) using the 18 capture probes for on-off KIR detection and 32,230 gene probes. The gene probes are 25mers and are detailed in a recent manuscript by Roe et al. that has been submitted for peer review and preprinted on bioRxiv (23). Synthetic probe matching was conducted via bbduk (24) with parameters " $\mathrm{k}=25$ maskmiddle $=\mathrm{f}$ overwrite $=\mathrm{t}$ rename $=\mathrm{t}$ nzo $=\mathrm{t}$ rcomp $=t$ ignorebadquality $=t$ ". This effectively removed any offKIR sequences and binned the sequences into 15 loci: 12 protein- coding genes, 2 pseudo-genes, and the intergenic region between KIR3DP1 and KIR2DL4. Sequences in each bin were de novo assembled with Canu 2.0 (25) (with default parameters except "genomeSize $=200 \mathrm{k}$ ") for each bin separately and all KIR sequences together (Figure 2D). The assemblies utilized only the captured sequences and were not assisted by any prior information, including individual genotypes or reference libraries.

\section{Step 7: Annotate the Assembled Sequences}

The capture probes were aligned to the haplotype-specific assembled sequences (i.e. haplotigs), and their patterns allowed gene-specific sequences to be extracted from the haplotypes (Figure 2E). The details are presented in the previouslymentioned preprint. At a high level, the algorithm uses the bowtie 2 alignment pattern of the 18 capture probes across the haplotigs/haplotypes to define locus-specific features. Within each feature, the locations of the exons, introns, and untranslated elements were located by searching for interelement boundaries with 16 base sequences as defined by the full haplotype MSA of 68 human haplotypes (Figure 2F). Each sequence contains 8 bases from one region and 8 from the other. For example, ACACGTGGGTGAGTCC spans the boundary between KIR2DL4's exon two and intron two; the first eight characters are from the second exon, and the last eight bases from the second intron. Boundary regions are flexible up to 3 mismatches if necessary. The locations of these elements allows for the annotation of protein, cDNA, and full-gene alleles with respect to names assigned in IPD-KIR. The haplotigs were ultimately annotated in GenBank's tbl format. BioJava (26) was used for some of the sequence processing. Reports on the assembly (or the raw sequences) were generated from Minimap2 (27), Qualimap (28), NanoPack (29), QUAST (30), Simple Synteny (31), and Tablet (32).

\section{Evaluation of the Workflow}

The capture-sequence-assemble workflow was evaluated on a cohort of 16 individuals whose haplotypes had previously been sequenced using fosmid separation and long-read sequencing $(13,33)$. The assembled haplotype-specific sequences (i.e., haplotigs), were evaluated by their phased coverage and concordance with the reference sequences as well as the number of haplotigs it takes to phase $75 \%$ of the haplotype (i.e., LG75). The LG metric is a standard metric for assembly evaluation; it is particularly appropriate for variable length haplotypes like KIR to evaluate their haplotigs by the fraction of the haplotype as opposed to number of bases. Of the 16 individuals, 8 are of European (EUR) ancestries (GenBank haplotype sequences KP420437-9, KP420440-6, KU645195-8, and KU842452) and 8 are of African American (AFA) ancestries (GenBank haplotype sequences MN167507, MN167510, MN167512, MN167513, MN167518, MN167519, and MN167520-9). The European haplotypes are detailed in Roe et al., 2017 (13), and the African Americans are detailed in the previously mentioned manuscript by Roe et al. that has been 
submitted for peer review. The distribution of haplotype structures in the European cohort is $8 \mathrm{cA} 01 \sim \mathrm{tA} 1$, and 1 each of $\mathrm{cA} 01 \sim \mathrm{tB} 01, \mathrm{cA} 01 \sim \mathrm{tB} 04, \mathrm{cA} 02 \sim \mathrm{tA} 03, \mathrm{cA} 03 \sim \mathrm{tB} 02, \mathrm{cB} 01 \sim \mathrm{tB} 01$, $c B 02 \sim t A 01$, and $c B 04 \sim t B 03$; one individual is homozygous for cA01 tA01 to within a few variants. The distribution of African American haplotypes is 5 cA01 tA01, 3 cB01 tA01, 3 cB03 tA01, 2 cB01 tB01, 1 cA01 tA02, 1 cA01 tB01, and 1 cB02 tA01. Further details are provided in Supplementary Table 1.

\section{Whole Genome Sequencing}

Theoretically, if KIR reads could be removed from WGS, the workflow should be able to assemble haplotypes the same as from targeted sequences. To test this hypothesis, whole genome CCS reads were obtained for an Ashkenazim individual (isolate NA24385) from the Genome In a Bottle (GIAB) consortium, as described in Wenger et al. (34). KIR ground truth was unknown previously. KIR reads were separated from WGS as described above and from there the workflow proceeded as usual from the error correcting step (Figure 2B).

\section{RESULTS}

Assemblies were evaluated with ground truth in 16 individuals (32 haplotypes) comprising 11 distinct haplotype structures. They were compared with the reference sequences shown in Figure 3, which depicts the 11 structures as connections between the same genes in different haplotypes and shows how the structures represent expansion and contraction of the A and $\mathrm{B}$ haplotype categories across the KIR3DP1-KIR2DL4 hotspot. Table 1 shows the results of the assembly compared with the reference sequences. For the 8 Europeans on average, the full set of 200 candidate probes provided $98 \%$ coverage, with $99.98 \%$ concordance, and it took 1.1 haplotigs to cover $75 \%$ of the reference (LG75). When a set of capture probes was reduced to a select 15 and evaluated on both cohorts, the European coverage lowered to $93 \%$, with the same concordance rate $(99.98 \%)$, and 1.3 LG75. The results for African Americans were very similar: $92 \%$ coverage, $99.98 \%$ concordance, and 1.6 LG75. When a select 3 more probes were added for a total of 18 capture probes, the assemblies for the 8 African Americans improved to $97 \%$ coverage, with $99.97 \%$ concordance, and 1.8 LG75. Most of the missing coverage occurred at the 3 ' end of the haplotypes: in certain KIR3DL2 alleles and some sequences extending 3' past KIR3DL2. Figure 4 shows the alignment of the haplotigs relative to the reference haplotype from the same individual MN167513 (cA01 tB01, Figure 3) in the 18-probe experiment. It shows a small $<2 \mathrm{~kb}$ gap in the assembly in KIR2DL3. Otherwise, the haplotigs provide complete and overlapping coverage across the reference haplotype sequence. When all the haplotigs are aligned the reference, the statistics report that it takes 2 haplotigs to assembly $75 \%$ of the haplotype and that total coverage is $98.4 \%$ with $99.98 \%$ concordance with the reference. The haplotigs are colored by base (ACGT) and indicate the haplotigs are concordant. Every gene is spanned by at least one haplotig, and all loci are phased with overlapping haplotigs, which the exception of the gap in KIR2DL3. Supplementary Figure 1 contains the assemblies for all individuals in all three sets of experiments, along with NanoPlot, Qualimap, and Quast reports. The reports contain different visualizations and collections of statistics like number and percentage of mapped/unmapped reads, $\mathrm{min} / \mathrm{max} / \mathrm{mean}$ read lengths, ACGT content, coverage, mapping quality, mismatch rates, and indel rates.

The optimized 18 capture probe provided results very similar to the full candidate set of 200 . Since this is the most efficient method, this probe coverage is further explained below. The 18 probes covered the haplotypes to an average distance of 2,398 bases. Figure 5 shows how the probes are distributed across a typical $19 \mathrm{~kb}$ region. The image shows an alignment displayed in Integrative Genomics Viewer (IGV) of the set of 18 probes to cB01 tB01 (GenBank reference KP420442). The top of the image shows it is zoomed into $49 \mathrm{~kb}$ of the haplotype $(\sim 50$ $100 \mathrm{~kb})$. In the middle track, the vertical ticks with the red numbers above indicate the alignment locations of the probes, with the red number being the label of the probe. In the bottom two tracks, the horizontal blue lines indicate the locations of exons (second from the bottom) and repetitive elements (bottom). The probe locations avoid the blue variable (exons) and repetitive (Alus, LINEs, etc.) regions but achieve complete coverage to a resolution of less than $5 \mathrm{~kb}$ across the $49 \mathrm{~kb}$. Only 7 distinct probes align to this region. From left to right, the probe sequence 4-3-12-10-2-7-13 occurs three times, except probe 10 does not align in the middle group. This alignment demonstrates how homology can be used to capture continuous KIR DNA over long distances with few probes without capturing off-KIR DNA. The set of 18 probes are included in Supplementary Table 2.

The CCS reads in the 18-probe experiment provided an average of $47 \mathrm{x}$ coverage of the haplotypes, except for a small gap in all alleles of KIR2DL2 and KIR2DL3, and a few alleles in other genes such as KIR2DS2. The gaps were on average 100 bases long, lead to gaps in the assembly $<2 \mathrm{~kb}$, and were most likely introduced during PCR amplification of a repeat-rich region. See the reports in Supplementary Figure 1 for more information.

Using the 18 sequences as virtual probes to capture KIR reads from WGS, the KIR assembled into a paternal cA01 tB01 $\left(\mathrm{KIR} 3 \mathrm{DL} 3{ }^{*} 00101 \sim \mathrm{KIR} 2 \mathrm{DL} 3{ }^{*} 00101 \sim \mathrm{KIR} 2 \mathrm{DP} 1{ }^{*} \mathrm{NEW} \sim\right.$ KIR2DL $1^{\star} 00302 \sim \mathrm{KIR} 3 \mathrm{DP} 1{ }^{\star} 0030202 \sim \mathrm{KIR} 2 \mathrm{DL} 4^{\star} 0050101 \sim$ KIR3DS $1^{*} 01301 \sim \mathrm{KIR} 2 \mathrm{DL} 5 \mathrm{~A}^{\star} 00101 \sim \mathrm{KIR} 2 \mathrm{DS} 5^{\star} 00201 \sim \mathrm{KI}$ $\left.\mathrm{R} 2 \mathrm{DS} 1^{\star} 00201 \sim \mathrm{KIR} 3 \mathrm{DL} 2^{\star} 00701\right)$ haplotype and a maternal c B $05 \sim$ tB 01 (KIR3 D L $3 * 00301 \sim$ KIR 2 D S $2 * 005 \sim$ KIR2DP $1{ }^{\star} \mathrm{NEW} \sim \mathrm{KIR} 2 \mathrm{DL} 1^{\star} 0040105 \sim \mathrm{KIR} 3 \mathrm{DP} 1^{\star} 0030202 \sim$ KIR2DL ${ }^{\star} 00501 \sim$ KIR3DS $1^{\star} 01301 \sim$ KIR2DL ${ }^{\star} 00101 \sim$ KIR2 DS5 $\left.5^{\star} 00201 \sim \mathrm{KIR} 2 \mathrm{DS} 1^{\star} 00201 \sim \mathrm{KIR} 3 \mathrm{DL} 2^{\star} \mathrm{NEW}\right)$; the assembly and its annotations are included in Supplementary Figure 2. The distal/ telomeric halves (KIR3DP1-KIR2DS1) are mostly homozygous, with approximately a dozen variants between them. It is possible this region is really deleted in maternal haplotype and should be classified as cB05 tB02 (KIR3DL3 KIR2DS2/KIR2DS3 KIR2DP1 KIR2DL1/KIR2DS2 KIR3DL2). Either way, this is the first reference haplotype with a $\mathrm{cB} 05$ with $\mathrm{tB} 01$ or $\mathrm{tB} 02$ in the same 


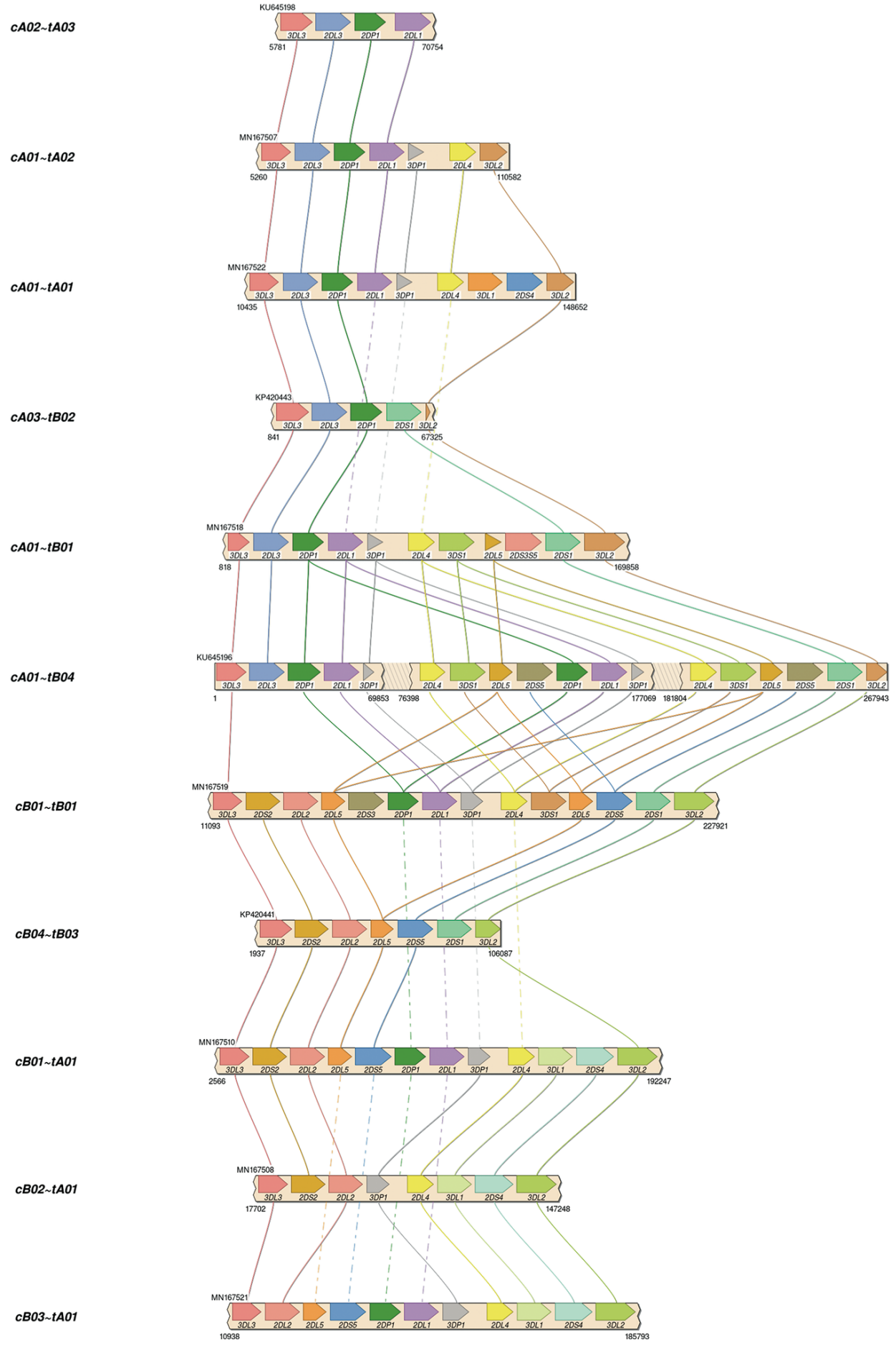

FIGURE 3 | Reference haplotype structures in the two validation cohorts. Each haplotype represents one of the structures previously established via fosmid library preparation and long-read sequencing. The unofficial name of the haplotype is on the left. Lines connect genes with the same name in different structures. Solid lines connect the same gene in neighboring structures. Dashed lines connect the same gene in non-neighboring structures (i.e., the line goes through one or more neighboring haplotypes). cA02 tA03: 1 EUR. cA01 tA02: 1 AFA. cA01 tA01: 5 AFA, 9 EUR. cA03 tB02: 1 EUR. cA01 tB01: 1 AFA, 1 EUR. cA01 tB04: 1 EUR. cB01 cB01: 2 AFA, 1 EUR. cB04 tB03: 1 EUR. cB01 tA01: 3 AFA. cB02 tA01: 1 EUR. cB03 tA01: 1 AFA. 
TABLE 1 | Assembly statistics.

\begin{tabular}{lcccc} 
pop. & probes \# & LG75 & coverage $\%$ & concordance \% \\
\hline EUR & 200 & 1.1 & $98 \%$ & $99.98 \%$ \\
EUR & 15 & 1.3 & $93 \%$ & $99.98 \%$ \\
AFA & 15 & 1.6 & $92 \%$ & $99.98 \%$ \\
AFA & $\mathbf{1 8}$ & $\mathbf{1 . 8}$ & $\mathbf{9 7 \%}$ & $\mathbf{9 9 . 9 7 \%}$ \\
\hline
\end{tabular}

For each of experiments in the rows, shown is the population, number of capture probes, the number of haplotigs spanning $75 \%$ of the haplotype (LG75), and the coverage of and concordance to the reference. The most efficient experiment is bolded.

haplotype; cB05 has KIR2DS2/KIR2DS3 fusion (named KIR2DS2*005 in IPD-KIR). The preprinted algorithm to genotype KIR from WGS confirms cB05 tB01 with cA01 tB01 (or their deleted forms) as the most-likely pair of structural haplotypes. The recently published PacBio full-genome assembly of this individual (34) assembled the cA01 tB01 in paternal haplotig SRHB01000968.1, but the maternal haplotigs do not contain any KIR haplotypes.

The code to assemble and annotate KIR haplotypes from CCS reads, including an example, is located at https://github.com/ droeatumn/kass. The "main" workflow performs the assembly. The "annotate" workflow labels the genes, exons, and introns in GenBank's.tbl format. The "align" workflow aligns the haplotigs to a reference and produces reports with which to evaluate the assembly or raw data. The code is supported by a Docker container at https:// hub.docker.com/repository/docker/droeatumn/kass, for convenient execution. The minimum recommended hardware for targeted sequencing is $30 \mathrm{G}$ main memory and $8 \mathrm{CPU}$ cores. More of each is helpful, especially with WGS. On an Ubuntu 18.04 Linux server with 40 core (Intel Xeon CPU E5-2470 v2 @ 2.40GHz) and 132G main memory, a single targeted assembly ( 70M fastq.gz) averaged 66 minutes and the WGS ( 70G fastq.gz) was 69 minutes. On MacOS 10.15.5 with 4 core (2.7 GHz Quad-Core Intel Core i7) and 16G main memory, a single targeted assembly averaged 125 minutes. Average times are reduced when assemblies are run in parallel.

\section{DISCUSSION}

These experiments in individuals from diverse populations demonstrate that KIR haplotypes can be efficiently enriched and ultimately assembled using an efficient number of capture probes. The workflow successfully reconstructed both haplotypes from targeted sequencing in 16 individuals and from WGS in 1 individual. Although recent advances in Single-Molecule RealTime sequencing by PacBio have improved quality and extended its applicability to WGS and highly repetitive regions, these advancements are not sufficient to accurately assemble KIR haplotypes. In our evaluations of self-reported results from other published and pre-published assemblers, we could not find another assembler that correctly assembled diploid KIR haplotypes from PacBio reads alone. The sequences need to be error corrected, separated from off-KIR, and sometimes the reads need to be separated and assembled on a per-gene basis depending on depth and genotypic variation, as more binning helps overcome the challenges of higher multiplexing. We have confirmed successful assembly with multiplexing up to eight individuals, which, we estimate, should lead to costs that rival full-exon short-read sequencing, and an order-of-magnitude

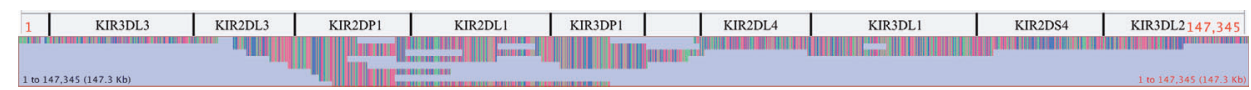

FIGURE 4 | Alignment of assembled haplotigs with reference haplotype sequence MN167513 (cA01 tA01), whose length is 147,345. The gene features are annotated across the top. The haplotigs are stacked below and colored by nucleotide.

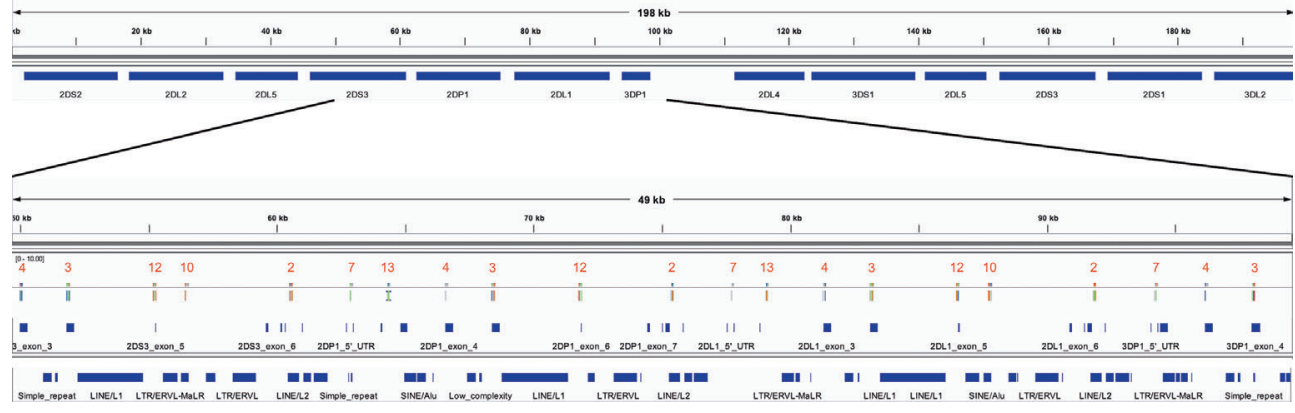

FIGURE 5 | IGV depiction of the alignment of the 18 set capture probes across a 49 kb region of KP420440 (cB01 tB01). The locations of the probes are displayed by the vertical ticks with the red labels above them in the middle track. The locations of exons and repeat elements (horizontal blue bars) are on the bottom two tracks. Seven distinct probes align in this window. The probe pattern 4-3-12-10-2-7-13 repeats three times from left to right, except probe 10 does not align in the middle group. 
more efficient than fosmid-based library preparation due to a more high-throughput library preparation workflow. We report efficiency details as a minimum, because the purpose of these experiments were to demonstrate the capability, not maximum efficiency. Our results suggested that lower bounds efficiency for off/on ratio for KIR capture range between 1.3 to 2.4 (Supplementary Table 3).

In addition to assembly, our software is the first annotation system for KIR haplotypes. The annotation algorithm leverages the information of the pattern of capture probes across the haplotype sequence to define loci and their exon/intron locations. Another system annotates gene alleles (35), but only if the input allele sequences start at the same location as their PCR primers.

There are 50 KIR reference haplotypes in the human genome reference GRCh38.p13 (36). The 16 African American haplotypes will be added in the next release, bringing the total to 66 . This is 3 times more than the entire chromosome 3 , which has the next highest total number of alternative haplotypes. Almost half of these KIR haplotype sequences were characterized in two workflows whose only common step was PacBio sequencing. Since both workflows agree over 99\%, we can be confident the cohorts have been characterized correctly and the two approaches validated each other.

A 2016 manuscript (37) describes PING, which is software to interpret KIR from short ( $<=300 \mathrm{bp})$ reads. It uses probes to capture 800 bp DNA fragments. Although the total number of probes required for KIR capture was unspecified, the total number for KIR and HLA was 10,456. PING's highest resolution results are obtained by aligning the short reads to full-gene references, calling the SNP variants, and then calling the two most likely reference alleles given the SNP genotypes. Although a great improvement over other current technologies at the time, the PING method does not phase/link variants within a gene or the haplotype as our long-read sequencing and capture method allows. Further, PING uses more than an order of magnitude more capture probes, which can be expensive to capture shorter fragments. It produces probabilistically phased lower-resolution predictions compared with our long-read assembly, which produces linked multi-gene and haplotype sequences without references. Therefore, we feel our method appreciably adds to the ability to properly analyze KIR regions by leveraging long read technologies for increased resolution and phasing compared with the previous NGS approach and also by leveraging high-throughput library preparation for reduced cost compared with the previous haplotyping approach.

In addition to demonstrating an efficient targeted haplotyping strategy, to the best of our knowledge, this the first report of KIR full diploid haplotype assembly from HiFi WGS alone. Our approach was able to assemble both haplotypes from WGS whereas the previously reported whole-genome assembly could not, underlying the necessity of a KIR-specific assembler. Both regions comprising the haplotype it missed (cB05 tB01 or a deleted form) are not in the primary human genome reference, and the two have not been reported together previously. Perhaps this lack of representation in the reference contributed to the missing assembly. Other possibilities include the lack of binning/ separation of KIR reads from the rest of the genome before assembling, or differences in the tools used in the workflow. Regardless, this experiment demonstrates the value added by the bioinformatics algorithms, in addition to the targeted capture and assembly.

The suspected amplification problem causing the small gap in KIR2DL2L3 occurs in a $\sim 100$ base region of poly-ATs, with L1s (and ALUs) on either side. It appears most or all PCR methods have a problem with this region, as almost every KIR2DL2 and KIR2DL3 reference allele has a different poly-AT sequence for this region (Supplementary Figure 3); these reference alleles were sequenced on various platforms but generally (if not fully) amplified with PCR. The PCR-less WGS from GIAB have no gaps, which suggests the source of the gap is PCR amplification and demonstrates that the assembler can correctly assemble this region along with the rest of the haplotypes when using non-PCR library preparation methods. Since KIR2DL2 or KIR2DL3 occur in most haplotypes and occur $\sim 10-20 \%$ from the proximal end, their short gap limits LG75 to its reported value of 1.8 in the AFA cohort. In cases where the gap is not an issue, such as WGS, LG75 will probably be 1, and LG100 will probably be a better metric.

The power of this method to assemble repetitive KIR regions without incorporating false non-KIR genomic signals may lie in the strongest recombination hotspot, the $10 \mathrm{~kb}$ intervening region between KIR3DP1 and KIR2DL4. Conventionally, KIR haplotype names (e.g., "cA01 tA01") have been described as two halves ("c" and " $\mathrm{t}$ ") separated by a recombination hotspot (“ $\sim$ "). The rate of recombination between the two halves is so frequent that any two may be found with each other, despite the relative evolutionary youth of the region. This hotspot stretches over $9 \mathrm{~kb}$ between KIR3DP1 and KIR2DL4. Any two alleles of this region are over $99 \%$ identical and consist of $13 \%$ Alus (SINEs) and $58 \%$ LINE1 repeat elements. Figure 6 shows an alignment of the region to itself. The top part of the figure displays the location of the KIR3DP1-KIR2DL4 intergenic region in the context of the cA01 tA01 primary human genome reference. The bottom half zooms into the intergenic region and shows a dot plot of the alignment. The lines on the dot plot indicate stretches of the haplotype that align with itself, either in the same location or a different location. The red lines indicate matching in the same orientation as the overall haplotype, and the blue indicate matching in the reverse complement. The red and blue horizontal bars at the bottom of the figure detail the location of repetitive elements. The red from $0-2,000$ simply shows that this region aligns with itself. There is a stretch of $3 \mathrm{~kb}$ from $\sim 5,200-8,200$ that reverse complement matches $\sim 400-3,400$. The yellow boxes highlight Alu repeats: AluSx3 from $\sim 800-1,000$ is matched in the reverse complement by AluSx1 $(\sim 6,400-6,600)$ and AluSx $4(\sim 7,100-7,400)$ and the same orientation by AluSq2 $(\sim 8,200-8,400)$. All elements are surrounded by very similar L1 elements for at least $1,000 \mathrm{bp}$ on both sides. This stretch of $3,000+$ bases of reverse complement repeats provides fertile ground for homologous recombination between the two halves of KIR haplotypes. This is the most difficult region to phase and 


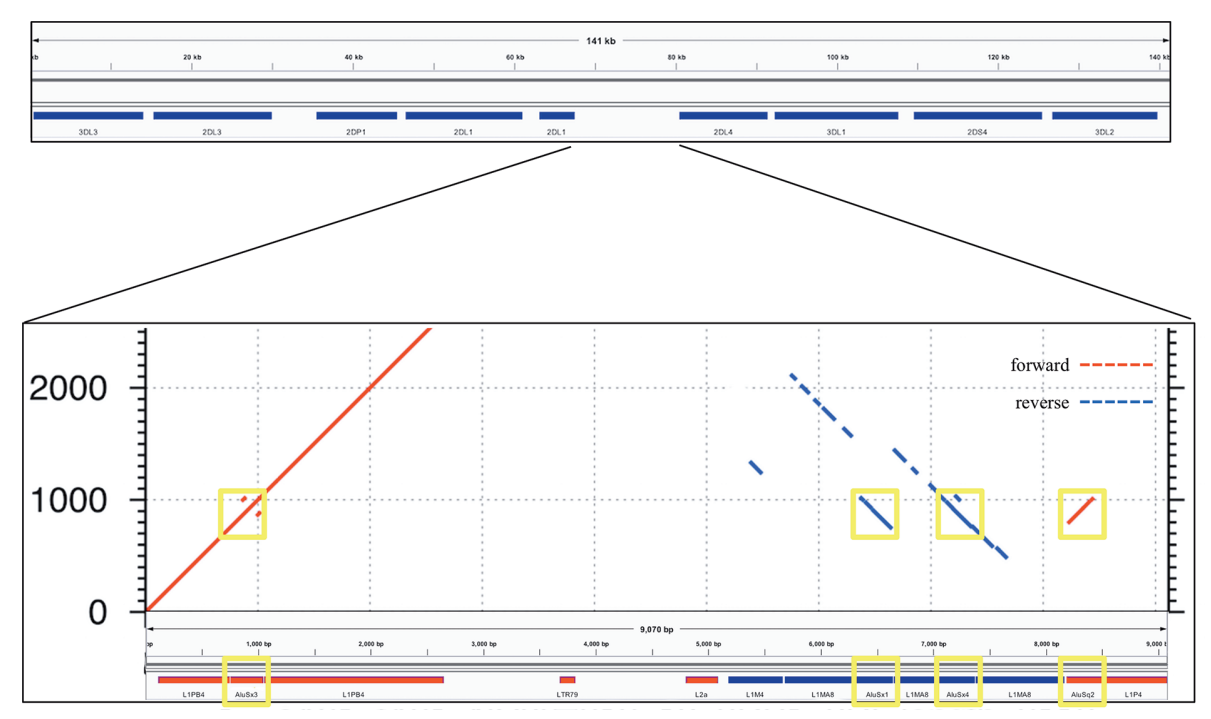

FIGURE 6 | Recombination hotspot. The top shows the $9 \mathrm{~kb}$ haplotype context of the repetitive region of the KIR3DP1-KIR2DL4 intergene region, whose selfalignment is shown in the bottom half. Red lines indicate alignment of the haplotype with itself in the same orientation, and blue lines indicate reverse complement orientation. The location of the repetitive elements is at the bottom, with red and blue again indicating orientation. The yellow boxes highlight three AluSx and one AluSq elements that align with each other, two in each direction.

the results demonstrate that the combination of variants and read length is generally high enough to phase full haplotypes with diploid reads. Although this region is an extreme example, the other recombination sites, which are usually internal to genes and result in gene fusions, homologously recombine via the same elements $(16,38-41)$, although only the KIR2DL4-KIR3DP1 intergenic region contains as many elements in both directions (10). Our assay is the only high-throughput method that allows analysis of all of these regions.

Future efforts include expanding testing to other populations, resolving the KIR2DL2L3 gap if possible, expanding capture for some KIR3DL2 alleles, expanding the assembly and annotation to bordering genes in the leukocyte receptor complex, optimizing multiplexing, and incorporate scaffolding into the workflow. Although the diverse AFA and EUR cohorts demonstrate proof of concept and expand our human genome references, it is important to develop reference sets for all populations. Expanding the capture would help ensure that KIR3DL3 and KIR3DL2 are sufficiently captured, help define any deleted haplotypes that may include these two genes and capture potentially relevant regulatory signals. Currently, all fully sequenced haplotypes contain some portion of these two bordering genes. "Scaffolding" is the term for combining haplotigs into one final haplotype sequence. Although the experiments revealed most haplotypes are covered in only two haplotigs and the haplotigs are simply subsequences of other haplotigs, it might help downstream analysis to include a rigorous scaffolding step; the KPI software may help select the appropriate references, as it can predict the pair of haplotype structures from the raw sequences.

Our approach leverages sequence similarity across multiple loci that were created by duplication followed by variation. Since this this is a true for many gene families, our approach should be more generally applicable to other regions that have a mix of homologous and variable/repetitive regions relative fragment length and capture characteristics.

The application of KIR genetics in medical research such as immunity, reproduction, and transplantation is encouraging, but limited by the technical difficulties for high-resolution interpretations at large scale and low cost. Here, a KIR haplotyping workflow was presented that can provide fullsequence haplotypes at approximately the same cost as full exon or full gene. For the first time, it allows high-resolution KIR haplotypes in population-sized cohorts, as opposed to lower-resolution genotypes. The analysis pipeline uses domain knowledge to assemble reads generated via well-established sequencing techniques that is accurate enough for personalized precision medicine and scalable to populations. To this point, most KIR association studies focus on variation at only one locus or one functional class to associate, while keeping the rest of the haplotypes static. Future full-haplotype studies will help KIR researchers better study gene combinations, regulatory regions, recombination hotspots, self-regulation, and non-binding factors that influence disease phenotypes. This increased ability will provide completed sets of population-specific reference haplotypes which will, among other things, enhance imputation power of lower resolution data. It allows for new comparisons that will provide insight into evolution and make this region the best annotated in the human genome, despite its complexity. Lastly, this novel approach will provide the capability to discover genetic associations in medically relevant areas such as infections, transplantation, cancer susceptibility, autoimmune diseases, reproductive conditions, and immunotherapy. The open and free software is available at 
https://github.com/droeatumn/kass and supported by a environment at https://hub.docker.com/repository/docker/ droeatumn/kass.

\section{DATA AVAILABILITY STATEMENT}

The datasets presented in this study can be found in online repositories. The names of the repository/repositories and accession number(s) can be found in the article/Supplementary Material or in the input directory at https:/github.com/ droeatumn/kass.

\section{ETHICS STATEMENT}

The studies involving human participants were reviewed and approved by National Marrow Donor Program Institutional Review Board. The patients/participants provided their written informed consent to participate in this study.

\section{AUTHOR CONTRIBUTIONS}

DR, JW, RK, and MM designed the experiments. DR, JW, KI, JB, $\mathrm{ND}$, and CL performed the experiments. All authors contributed to the article and approved the submitted version.

\section{FUNDING}

Supported by a grant from the Department of the Navy, Office of Naval Research (N00014-19-1-2888).

\section{REFERENCES}

1. Wroblewski EE, Parham P, Guethlein LA. Two to Tango: Co-evolution of Hominid Natural Killer Cell Receptors and MHC. Front Immunol (2019) 10:177. doi: 10.3389/fimmu.2019.00177

2. Rajalingam R. Human diversity of killer cell immunoglobulin-like receptors and disease. Korean J Hematol (2011) 46:216. doi: 10.5045/kjh.2011.46.4.216

3. Martin MP, Qi Y, Gao X, Yamada E, Martin JN, Pereyra F, et al. Innate partnership of HLA-B and KIR3DL1 subtypes against HIV-1. Nat Genet (2007) 39:733-40. doi: 10.1038/ng2035

4. Davis C, Rizzieri D. Immunotherapeutic Applications of NK Cells. Pharmaceuticals (2015) 8:250-6. doi: 10.3390/ph8020250

5. Foley B, Felices M, Cichocki F, Cooley S, Verneris MR, Miller JS. The biology of NK cells and their receptors affects clinical outcomes after hematopoietic cell transplantation (HCT). Immunol Rev (2014) 258:45-63. doi: 10.1111/ imr.12157

6. Benson DM, Caligiuri MA. Killer Immunoglobulin-like Receptors and Tumor Immunity. Cancer Immunol Res (2014) 2:99-104. doi: 10.1158/23266066.CIR-13-0219

7. Moffett A, Colucci F. Co-evolution of NK receptors and HLA ligands in humans is driven by reproduction. Immunol Rev (2015) 267:283-97. doi: 10.1111/imr.12323

8. Leaton LA, Shortt J, Kichula KM, Tao S, Nemat-Gorgani N, Mentzer AJ, et al. Conservation, Extensive Heterozygosity, and Convergence of Signaling

\section{ACKNOWLEDGMENTS}

This manuscript has been released as a pre-print at bioRxiv with the same title and authors (42). Thanks to Cynthia Vierra-Green from CIBMTR, Julia Udell from BICB UMN, and Richard Hall from PacBio for advice and review.

\section{SUPPLEMENTARY MATERIAL}

The Supplementary Material for this article can be found online at: https://www.frontiersin.org/articles/10.3389/fimmu.2020. 582927/full\#supplementary-material

SUPPLEMENTARY FIGURE 1 | AFA and EUR haplotigs. Data Sheets 2-5 are zip files containing the assembled haplotigs for all AFA and EUR assemblies. Also included are Qualimap, NanoPack, and QUAST reports.

SUPPLEMENTARY FIGURE 2 | WGS haplotigs. Data Sheet 1 is a zipped file containing the assembled haplotigs for maternal and paternal haplotypes for WGS assembly from GIAB individual NA24385.

SUPPLEMENTARY FIGURE 3 | Image 3.tif contains a multiple sequence alignment of reference KIR2DL2 alleles in IPD-KIR. The variation between columns 6,706 and 6,744 demonstrates extensive reported variation in a poly-AT region.

SUPPLEMENTARY TABLE 1 | Cohort details. In Table 1, the first column contains the individual ID used for this study. The second column contains the GenBank accession number of the reference haplotype. Accessions that start with $\mathrm{K}$ are from the European cohort, and accessions that start with $\mathrm{M}$ are form the African American cohort. The third column contains the informal haplotype name.

SUPPLEMENTARY TABLE 2 | Capture probes. Data sheet 6 contains the 18 capture probe sequences in zipped fasta format.

SUPPLEMENTARY TABLE 3 | The spreadsheet Table 2 contains statistics calculating the amount and ratios of on and off KIR sequences in the experiments that differ by cohort and number of probes.

Potential All Indicate a Critical Role for KIR3DL3 in Higher Primates. Front Immunol (2019) 10:24. doi: 10.3389/fimmu.2019.00024

9. IPD-KIR. https://www.ebi.ac.uk/ipd/kir/.

10. Wilson MJ, Torkar M, Haude A, Milne S, Jones T, Sheer D, et al. Plasticity in the organization and sequences of human KIR/ILT gene families. Proc Natl Acad Sci (2000) 97:4778-83. doi: 10.1073/pnas.080588597

11. Pyo C-W, Guethlein LA, Vu Q, Wang R, Abi-Rached L, Norman PJ, et al. Different Patterns of Evolution in the Centromeric and Telomeric Regions of Group A and B Haplotypes of the Human Killer Cell Ig-Like Receptor Locus. PloS One (2010) 5:e15115. doi: 10.1371/journal.pone.0015115

12. Pyo C-W, Wang R, Vu Q, Cereb N, Yang SY, Duh F-M, et al. Recombinant structures expand and contract inter and intragenic diversification at the KIR locus. BMC Genomics (2013) 14:89. doi: 10.1186/1471-2164-14-89

13. Roe D, Vierra-Green C, Pyo C-W, Eng K, Hall R, Kuang R, et al. Revealing complete complex KIR haplotypes phased by long-read sequencing technology. Genes Immun (2017) 18(3):127-34. doi: 10.1038/gene.2017.10

14. Schwartz JC, Gibson MS, Heimeier D, Koren S, Phillippy AM, Bickhart DM, et al. The evolution of the natural killer complex; a comparison between mammals using new high-quality genome assemblies and targeted annotation. Immunogenetics (2017) 69:255-69. doi: 10.1007/s00251-017-0973-y

15. Jiang W, Johnson C, Jayaraman J, Simecek N, Noble J, Moffatt MF, et al. Copy number variation leads to considerable diversity for B but not A haplotypes of the human KIR genes encoding NK cell receptors. Genome Res (2012) 22:1845-54. doi: 10.1101/gr.137976.112 
16. Traherne JA, Martin M, Ward R, Ohashi M, Pellett F, Gladman D, et al. Mechanisms of copy number variation and hybrid gene formation in the KIR immune gene complex. Hum Mol Genet (2010) 19:737-51. doi: 10.1093/hmg/ ddp538

17. Uhrberg M, Valiante NM, Shum BP, Shilling HG, Lienert-Weidenbach K, Corliss B, et al. Human Diversity in Killer Cell Inhibitory Receptor Genes. Immunity (1997) 7:753-63. doi: 10.1016/S1074-7613(00)80394-5

18. Robinson J, Halliwell JA, Hayhurst JD, Flicek P, Parham P, Marsh SGE. The IPD and IMGT/HLA database: allele variant databases. Nucleic Acids Res (2014) 43(1):D423-31. doi: 10.1093/nar/gku1161

19. RepeatMasker. http://www.repeatmasker.org/.

20. Bao W, Kojima KK, Kohany O. Repbase Update, a database of repetitive elements in eukaryotic genomes. Mobile DNA (2015) 6:11. doi: 10.1186/ s13100-015-0041-9

21. Multiplex Genomic DNA Target Capture Using IDT xGen ${ }^{\circledR}$ Lockdown ${ }^{\circledR}$ Probes. Available at: https://www.pacb.com/wp-content/uploads/ProcedureChecklist-\%E2\%80\%93-Multiplex-Genomic-DNA-Target-Capture-UsingIDT-xGen-Lockdown-Probes.pdf (Accessed August 31, 2020).

22. Salmela L, Walve R, Rivals E, Ukkonen E. Accurate self-correction of errors in long reads using de Bruijn graphs. Bioinformatics (2016) 799-806. doi: 10.1093/bioinformatics/btw321

23. Roe D, Kuang R. Accurate and Efficient KIR Gene and Haplotype Inference from Genome Sequencing Reads with Novel K-mer Signatures. bioRxiv (2019). doi: 10.1101/541938

24. BBTools. . http://sourceforge.net/projects/bbmap/.

25. Koren S, Walenz BP, Berlin K, Miller JR, Bergman NH, Phillippy AM. Canu: scalable and accurate long-read assembly via adaptive k-mer weighting and repeat separation. Genome Res (2017) 27:722-36. doi: 10.1101/gr.215087.116

26. Lafita A, Bliven S, Prlić A, Guzenko D, Rose PW, Bradley A, et al. BioJava 5: A community driven open-source bioinformatics library. PloS Comput Biol (2019) 15:e1006791. doi: 10.1371/journal.pcbi.1006791

27. Li H. Minimap2: pairwise alignment for nucleotide sequences. Bioinformatics (2018) 34:3094-100. doi: 10.1093/bioinformatics/bty191

28. Okonechnikov K, Conesa A, García-Alcalde F. Qualimap 2: advanced multisample quality control for high-throughput sequencing data. Bioinformatics (2015) 292-4. doi: 10.1093/bioinformatics/btv566

29. De Coster W, D'Hert S, Schultz DT, Cruts M, Van Broeckhoven C. NanoPack: visualizing and processing long-read sequencing data. Bioinformatics (2018) 34:2666-9. doi: 10.1093/bioinformatics/bty149

30. Gurevich A, Saveliev V, Vyahhi N, Tesler G. QUAST: quality assessment tool for genome assemblies. Bioinformatics (2013) 29:1072-5. doi: 10.1093/ bioinformatics/btt086

31. Veltri D, Wight MM, Crouch JA. SimpleSynteny: a web-based tool for visualization of microsynteny across multiple species. Nucleic Acids Res (2016) 44:W41-5. doi: 10.1093/nar/gkw330

32. Milne I, Stephen G, Bayer M, Cock PJA, Pritchard L, Cardle L, et al. Using Tablet for visual exploration of second-generation sequencing data. Briefings Bioinf (2013) 14:193-202. doi: 10.1093/bib/bbs012

33. Roe D, Vierra-Green C, Pyo C-W, Geraghty DE, Spellman S, Kuang R, et al. A Detailed View of KIR Haplotype Structures and Gene Families as Provided by a New Motif-based Multiple Sequence Alignment. bioRxiv (2020). doi: 10.1101/2020.08.07.242305

34. Wenger AM, Peluso P, Rowell WJ, Chang P-C, Hall RJ, Concepcion GT, et al. Accurate circular consensus long-read sequencing improves variant detection and assembly of a human genome. Nat Biotechnol (2019) 37:1155-62. doi: 10.1038/s41587-019-0217-9

35. Surendranath V, Albrecht V, Hayhurst JD, Schöne B, Robinson J, Marsh SGE, et al. TypeLoader: A fast and efficient automated workflow for the annotation and submission of novel full-length HLA alleles: SURENDRANATH et al. HLA (2017) 90:25-31. doi: 10.1111/tan.13055

36. KIR haplotypes in the human genome reference. Genome Reference Consortium. (2020). Available at: https://www.ncbi.nlm.nih.gov/grc/human? filters=chr:19+gene:KIR\#current-regions.

37. Norman PJ, Hollenbach JA, Nemat-Gorgani N, Marin WM, Norberg SJ, Ashouri E, et al. Defining KIR and HLA Class I Genotypes at Highest Resolution via High-Throughput Sequencing. Am J Hum Genet (2016) 99:375-91. doi: 10.1016/j.ajhg.2016.06.023

38. Martin AM, Freitas EM, Witt CS, Christiansen FT. The genomic organization and evolution of the natural killer immunoglobulin-like receptor (KIR) gene cluster. Immunogenetics (2000) 51:268-80. doi: 10.1007/s002510050620

39. Martin MP, Bashirova A, Traherne J, Trowsdale J, Carrington M. Cutting Edge: Expansion of the KIR Locus by Unequal Crossing Over. J Immunol (2003) 171:2192-5. doi: 10.4049/jimmunol.171.5.2192

40. Sambrook JG. Single haplotype analysis demonstrates rapid evolution of the killer immunoglobulin-like receptor (KIR) loci in primates. Genome Res (2005) 15:25-35. doi: 10.1101/gr.2381205

41. Guethlein LA, Older Aguilar AM, Abi-Rached L, Parham P. Evolution of Killer Cell Ig-Like Receptor ( KIR ) Genes: Definition of an Orangutan KIR Haplotype Reveals Expansion of Lineage III KIR Associated with the Emergence of MHC-C. J Immunol (2007) 179:491-504. doi: 10.4049/ jimmunol.179.1.491

42. Roe D, Williams J, Ivery K, Brouckaert J, Downey N, Locklear C, et al. Efficient Sequencing, Assembly, and Annotation of Human KIR Haplotypes. bioRxiv (2020). doi: 10.1101/2020.07.12.199570

Conflict of Interest: JW, KI and JB were employed by Laboratory Corporation of America Holdings. ND and CL were employed by Integrated DNA Technologies Inc.

The remaining authors declare that the research was conducted in the absence of any commercial or financial relationships that could be construed as a potential conflict of interest.

Copyright (C) 2020 Roe, Williams, Ivery, Brouckaert, Downey, Locklear, Kuang and Maiers. This is an open-access article distributed under the terms of the Creative Commons Attribution License (CC BY). The use, distribution or reproduction in other forums is permitted, provided the original author(s) and the copyright owner(s) are credited and that the original publication in this journal is cited, in accordance with accepted academic practice. No use, distribution or reproduction is permitted which does not comply with these terms. 